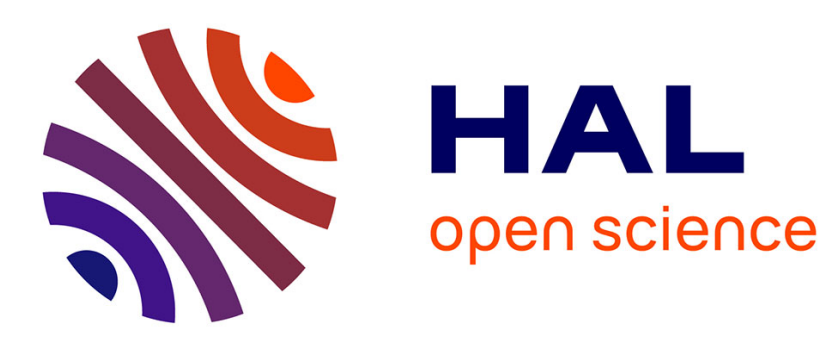

\title{
A Modeling Framework for Efficacy Assessment and Preventive Maintenance of Torrential Protection Works
}

Nour Chahrour, Sleiman Hariri, Jean-Marc Tacnet, Christophe Bérenguer

\section{To cite this version:}

Nour Chahrour, Sleiman Hariri, Jean-Marc Tacnet, Christophe Bérenguer. A Modeling Framework for Efficacy Assessment and Preventive Maintenance of Torrential Protection Works. ESREL 2019 - 29th European Safety and Reliability Conference, Sep 2019, Hannover, Germany. pp.444-451, 10.3850/978981-11-2724-3_0327-cd. hal-02318144

\section{HAL Id: hal-02318144 \\ https://hal.science/hal-02318144}

Submitted on 19 Oct 2019

HAL is a multi-disciplinary open access archive for the deposit and dissemination of scientific research documents, whether they are published or not. The documents may come from teaching and research institutions in France or abroad, or from public or private research centers.
L'archive ouverte pluridisciplinaire HAL, est destinée au dépôt et à la diffusion de documents scientifiques de niveau recherche, publiés ou non, émanant des établissements d'enseignement et de recherche français ou étrangers, des laboratoires publics ou privés. 


\title{
A Modeling Framework for Efficacy Assessment and Preventive Maintenance of Torrential Protection Works
}

\author{
Nour Chahrour \\ Univ. Grenoble Alpes, Irstea, ETNA, 38000 Grenoble, France. E-mail: nour.chahrour@irstea.fr \\ Sleiman Hariri \\ Univ. Grenoble Alpes, Irstea, ETNA, 38000 Grenoble, France.E-mail: sleiman.hariri@irstea.fr \\ Jean-Marc Tacnet \\ Univ. Grenoble Alpes, Irstea, ETNA, 38000 Grenoble, France.E-mail: jean-marc.tacnet@irstea.fr \\ Christophe Bérenguer \\ Univ. Grenoble Alpes, CNRS, Grenoble INP*, GIPSA-lab, 38000 Grenoble, France. \\ E-mail: christophe.berenguer@grenoble-inp.fr
}

Natural phenomena in mountains put people and assets at risk. Risk reduction measures can be either structural (protection works) or non-structural (risk zoning maps). In torrential watersheds of the French mountains, many checkdams have been built since the $19^{t h}$ century. As any civil engineered structure, those dams age and their failures may have severe effects on protected areas. Thus, preserving their level of efficacy is of a high interest. In a context of decreasing public budgets, it is necessary to assess their structural, functional and economic efficacy in order to quantify the residual risk and to choose the best maintenance strategies. Recent global approach has been proposed to integrate safety and reliability analysis, multicriteria decision-making methods, and information imperfection processing. However, it does not help in choosing the best strategy to maintain protection devices. It does not cover all aspects related to protection works management making the balance between investment, preventive maintenance costs, and risk evolution. This paper develops a contribution addressing all those issues and proposes a new modeling approach based on Petri nets, whose main steps are: 1) to describe multi-scale protection works systems interaction between both natural and technological systems' components; 2) to analyze structural and functional failure modes; 3 ) to develop a Petri net model for deterioration and maintenance modeling; 4) to compare different maintenance strategies under different hypothesis on degradation and damage processes.

Keywords: Preventive maintenance, deterioration modeling, decision-making, Petri nets, protection structures, checkdams.

\section{Introduction}

Mountainous regions, e.g. French Alpes, are usually exposed to different types of natural phenomena such as torrential floods, debris flows, landslides, etc. These hazards will pose a threat to humans and to vulnerable assets resulting in direct destruction and indirect economical damages. The magnitude and the sudden effects of natural phenomena push to acquire a comprehensive knowledge about their dynamics as well as means and alternatives for protecting people and properties. In mountain streams, since the $19^{\text {th }}$ century, engineers have tried to reduce the risk generated from torrential activities by constructing a series of torrential protection works along the watershed. Among all civil engineering structures, checkdams are perhaps the most used. Although protection structures aim in risk prevention and mitigation, yet, they are considered as critical structures because they constitute a complex system that age, deteriorate, and may be damaged overtime with a high level of induced consequences. As the level of deterioration of protection works increases, their performance level decreases and the risk is no more reduced as much as it should be.

Assessing the efficacy of protection structures is linked to the efficacy expected at the design phase, after construction, and in service Carladous (2017). Moreover, it involves three aspects Tacnet et al. (2016): structural, functional, and economical efficacy. Consequently, evaluating the efficacy of protection structures requires inspection visits conducted regularly at defined time intervals. Inspections are based on indicators ex-

Proceedings of the 29th European Safety and Reliability Conference.

Edited by Michael Beer and Enrico Zio

Copyright (C) 2019 by ESREL2019 Organizers. Published by Research Publishing, Singapore

ISBN: 981-973-0000-00-0 :: doi: 10.3850/981-973-0000-00-0_esrel2019-paper 
tracted from pathologies and evaluated by visual observation.

Interdependencies between failures may occur either within the same component or within the different components of the system (cascade failure Kotzanikolaou et al. (2013)). To avoid the failure of a complete system of protection works, maintenance of these structures must be ensured over time. Maintenance operations aim in improving the quality, reliability, safety, and dependability of a system Arab Maki and Shariat Zadeh (2010). Two types of maintenance can be distinguished: preventive maintenance, applied before the failure of the system and corrective maintenance (replacement), carried out after the failure of the system. In order for decision-makers to choose between several alternatives to be applied to a system, it is essential to monitor these structures during their lifetime period and to model their evolution from one state to another when they are exposed to deterioration mechanisms or maintenance strategies. Presently, decisionmaking in the domain of maintenance management plays a significant role in progressing productivity efficiency and in enhancing maintenance budget Sabaei et al. (2015).

Dependability analysis starts by defining all the functions of the system, analyzing all the possible failure modes and proposing various procedures to reduce the associated risk. Reliability is one aspect of dependability. It is defined as the ability of the system to operate under certain conditions and for a specified period of time. It is essential to model the interdependencies between the various components that constitute the system in order to determine the overall system reliability. Indeed, several methods Modarres (1992) are used for this purpose such as fault tree analysis, event tree analysis, failure mode and effect analysis, etc. The main idea is to represent graphically the logic of a dysfunction of a system due to an occurring event. All these methods are used to predict the performance of the structure, take decisions, and to mitigate failure.

In fact, several maintenance strategies and solutions can be carried out on a system when exposed to a certain failure. However, with the help of decision-aiding models, decision-makers can justify rigorously the choice of which strategy to follow. Cost-benefit analysis is the most widely used decision support method for assessing the economic component of system efficacy. Yet, the choice of the model to be applied on a given system should be based on accepting certain hypothesis, principals, and assumptions. In the present study, decision-aiding models based on Petri nets will be presented. These models are able to describe the behavior of the system during its lifetime period. Petri nets offer a flexible and efficient method to model the deterioration and maintenance process of structures. It has the capacity to model the reality compared to other developed models Le and Andrews (2016).

In the next section, a brief description of Petri net models is provided. The third section presents the proposed structure of the Petri net model that can be applied to any system. The proposed model is then applied in the fourth section for modeling the behavior of checkdams when exposed to functional and structural deterioration and to maintenance operations. In the fifth section, the results obtained after simulating the model are discussed. Finally, the conclusion summarizes the work achieved and suggestions for further developments.

\section{Petri Nets (PNs)}

PNs were developed by the German Carl Adam in the 1962 Adam (1962). Since then, they have been developed and extended to be applied for railway networks Andrews (2013) and bridge risk assessment Le et al. (2017). PNs are graphical tools similar to the directed bipartite graphs that can be simulated to analyze existing processes arising in systems with different components.

\subsection{PNs elements}

PNs consist of four different elements: places, tokens, transitions and arcs. A place, symbolized by a circle, corresponds to a state of the system and represents a certain condition. A token, denoted by a filled solid small circle, is located in a place to indicate the state of the system at a given time. A transition, symbolized by a rectangle, is responsible of moving tokens from a place to another due to an event which leads to the change of state in the system. Arcs, represented by arrows, connect places to transitions and vice versa. The direction of the arc represents the input or output place of the transition. Each arc is characterized by a certain multiplicity given by a natural number. When the multiplicity of an arc is not indicated, it is by default equals to one.

\subsubsection{PNs operation}

- Transition firing: a transition is enabled if and only if each of its input places is marked by a number of tokens at least equal to the multiplicity of the arc connecting it to the transition. Once the transition is enabled, it will be fired removing tokens from the input places and depositing tokens in the output places according to their arc multiplicities.

- Transition firing time: it represents the residence time of a token in a place (sojourn time). It is a necessary data that control the simulation of the PN model. The determination of firing delay time can be either given deterministically (Deterministic Timed PNs) or probabilistically (Stochastic Timed PNs). 


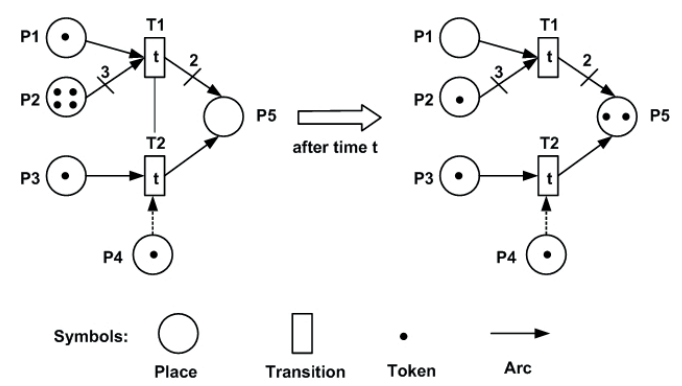

Fig. 1. Simple PN illustrating firing process and inhibitor arc

- Inhibitor arc: additional form of arcs represented by dashed arc and can be only directed from a place to a transition. It aims to keep the transition disabled inhibiting it from firing. This is accomplished when the multiplicity of the inhibitor arc is equal to the number of tokens in the input place.

A simple PN example is illustrated in Fig. 1. The number of tokens in the input places P1, P2 and P3 $(1,4,1)$ of transitions $\mathrm{T} 1$ and $\mathrm{T} 2$ is equal or above the arcs multiplicity of each $(1,3,1)$. Hence, T1 and T2 are enabled. However, the token in place $\mathrm{P} 4$ inhibits the firing of transition $\mathrm{T} 2$. Therefore, after time $\mathrm{t}, \mathrm{T} 1$ is only fired, and a number of tokens equal to the multiplicity of each arc is removed from the input places of $\mathrm{T} 1$ and deposited to the output places of T1.

\subsubsection{Stochastic Petri nets SPNs}

SPNs follow probabilistic models on transitions based on historical records and data used for dependability modeling. Stochastic models are superior to other modeling techniques because they offer practicality and reliability Yianni et al. (2017).

In dependability assessment, stochastic variables are time variables such as the time taken by a component to fail or to be repaired Aubry et al. (2016). This reveals that the crucial elements which are able to represent the behavior of the system are the transition firing times. SPNs are thus specialized by the occurrence of an event characterized by a rate giving the time needed for the transition to be fired. In the presence of such variables, their will be no conflict between the firing of the transitions since the fired transition will be the one whose associated event occurs first.

Several probability distributions are used in reliability analysis. Exponential distribution is usually used for its simplicity because it considers a constant failure rate. However, some researchers considered that constant failure rates are rarely appropriate for deterioration modeling Le and Andrews (2016). This refers to the fact that failure process represented by exponential distribution cannot model the aging of the system.

\section{PN Modeling Structure}

This section presents the global framework of the PN model able to describe the evolution of the state of a system when exposed to degradation processes or to maintenance actions. The model permits to compare the maintenance strategies in terms of efficiency and cost.

The PN model is constructed using GRIF (2017) software developed by TOTAL. The GRIF package related to PN modeling, uses the MOCARP computation engine, based on Monte Carlo simulation, which is generally used for evaluating the reliability of a complex system Sava et al. (2015). After each simulation, the sojourn time in each state and number of occurrence of different events can be obtained.

\subsection{Deterioration process}

A gradual deterioration model is based on the existence of a degradation indicator, able to provide information about the degradation level of the system at a specified time. In fact, each deterioration mechanism corresponds to a degradation indicator which reveals the state of the system. Hence, this model is required for the determination of the number of places in the PN. In this model, it is considered, based on an expert that the system resides in one of the four states: new, poor, very poor and failed.

An example of a net modeling the deterioration process is represented in Fig. 2. The four states describing the evolution of the system are represented by places: P1 - P4. P1 corresponds to the new state of the system, P2 and P3 are the intermediate states, and $\mathrm{P} 4$ represents the failure of the system. Transitions T1 - T3 link between the degraded states. The firing delay times corresponding to these transitions should be given as probability distributions based on historical data or on assumptions made by experts.

At $t=0$, a token is placed in $\mathrm{P} 1$ revealing that, at the initial state, the system is considered to be new.

\subsection{Inspection process}

The state of the system will only be detected following an inspection. The system will be inspected periodically. Inspections will take place at a determined regular time interval $t_{\text {insp }}$ that corresponds to the firing of the transition T5.

At $t=0$, an additional token is added to $\mathrm{P} 5$. When the firing delay time $t_{i n s p}$ of transition T5 is reached, the token travels to P6 in which inspection takes place. Consequently, one of the immediate transitions T6, T7 or T8 will immediately fire if a token exists respectively in P2, P3 or P4. In addition, T4 will fire and the token will 


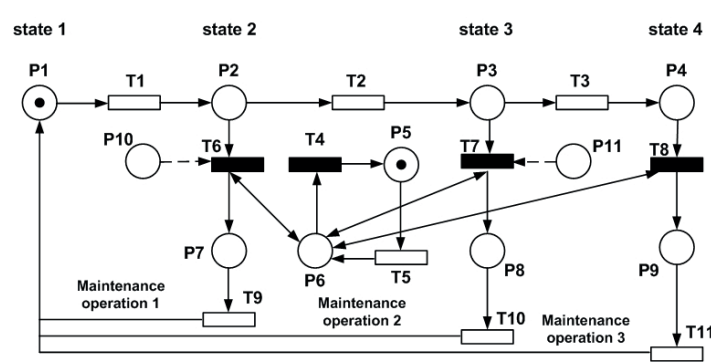

Fig. 2. An example of Petri net model (S.Hariri)

return to P5 waiting for another inspection to take place after a period of time $t_{\text {insp }}$.

When the system is in state 2 , a token is present in $\mathrm{P} 2$ and two possible pathways exist. If transition T6 is fired first, the token will move to P7 meaning that the system is inspected and a maintenance operation will be applied. However, if transition T2 is fired first, the token will travel to $\mathrm{P} 3$ which means the system has degraded to state 3 before being inspected. Similarly, if the system is in state 3 , a token is present in P3 and may either move to P8 (T7 is fired first) where the system is inspected and a maintenance operation will be applied or it moves to the failed state P4 (T3 is fired first) before being inspected. If the system is in state 4, a token is present in $\mathrm{P} 4$ and only T8 can be fired. The system will wait for inspection to occur and a maintenance operation will be applied.

\subsection{Maintenance process}

When the condition or the state of the system is identified, the appropriate type of maintenance operation can be applied to restore the system to its initial state. A condition-based maintenance policy is applied in this model in which each state will correspond to a maintenance operation. Places P7 - P9 represent the states revealed after inspection. When a token is present in $\mathrm{P} 7, \mathrm{P} 8$ or $\mathrm{P} 9$, this indicates that maintenance operation 1,2 or 3 is required respectively. Following maintenance, transition T9, T10 or T11 fires and moves the token to P1. Transitions T9 - T11 represent the repair planning time for each maintenance operation, which is the time needed to schedule and carry out the work.

Maintenance operations presented in this model are considered perfect. The system is restored to the initial new state after the application of each maintenance operation.

\subsection{Comparison of strategies}

The addition of places P10 - P11, associated with inhibitor arcs, permits the simulation of different maintenance strategies. If P10 is marked with a token, T6 is inhibited and will not fire as long as the token in P10 remains. Consequently, over the lifetime period of the system, maintenance operation 1 cannot take place and the system is allowed to degrade further to state 3 . Similarly, different strategies can be considered by adding a token to P11 for example, where maintenance operation 2 cannot be carried out.

After simulation, the model provides the number of maintenance operations applied in each strategy. Therefore, knowing the cost of each maintenance operation, the total cost of each maintenance strategy can be computed. As mentioned before, the model is able to provide the sojourn time of the system in each state. These outcomes can be adequate for making a decision regarding the most suitable maintenance strategy to be applied to the system.

\section{Application on Checkdams}

This application is selected in order to illustrate the capabilities of the modeling approach to evaluate different maintenance strategies. Checkdams, as critical structures, show complexities in relating possible failures and indicators responsible for their evolution. Complexity is also related to the fact that a system can be composed of several components interacting with each other to accomplish the systems functions. The main objective of the approach developed is to show that the methodology coming more from industrial issue can support decision-making.

Checkdams aim to reduce the causes that leads to the occurrence of risks generated from mountains. Their main functions are torrent bed stabilization, slope reduction, prevention of longitudinal erosion, and flow centering. However intense waterfall evacuated by the center of the hydraulic section of checkdams results in scouring under the center of their foundations. This phenomenon affects the performance level of checkdams in fulfilling their functions. The level of scouring will increase over time and may reach critical levels in which failure may occur. The present study aims to investigate the stability of checkdams when confronting the risk of scouring and then to choose the most suitable maintenance strategy.

Scouring is considered as a functional failure which will then lead to a structural failure by affecting the external stability of the checkdam. As the level of scouring increases, the structure will be subjected to tilting. In this case, if no intervention is made, the structure will continue to tilt more until it fails by overturning which is considered as a structural failure. Fig. 3 represents the behavior of the system in four different states. Each state is a combination of a functional state (FS) and a structural state (SS).

The interaction between structural and functional states evolutions is actually linked to the 


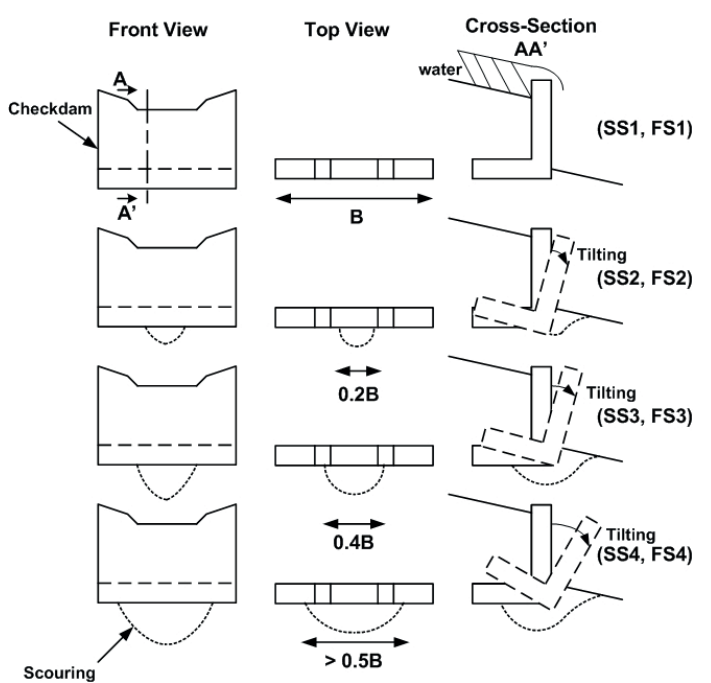

Fig. 3. Degradation states due to scouring of a checkdam

amount of scouring under the foundation of the checkdam. It is assumed that if $20 \%$ of the foundations' width is scoured, the structure will start to tilt. As the scour level increases, the structure will tilt more and more until it completely fails by overturning when more than half of the foundations' width is scoured. However, structural deterioration may be also triggered by other factors such as structural behavior. For example, intense deposition increases the lateral pressure thus affecting the stability of the checkdam.

\subsection{Extended PN model}

For modeling the stability of a checkdam, structural deterioration is modeled taking into account its dependability with functional deterioration. The model has an extended structure of that developed in Section 3.1 in which the interactions between the different failure modes are considered in addition to inspection and maintenance steps. Fig. 4 represents the extended PN model applied for analyzing the effect of functional deterioration on the structural stability of the system allowing the simulation of different maintenance strategies.

Four functional degradation states are defined: FS1, FS2, FS3, and FS4 corresponding to 0\%, $20 \%, 40 \%$, and $50 \%$ scoured foundation respectively. It is assumed that each of these states influence the structural state of the structure. Thus four structural states are also defined as SS1, SS2, $\mathrm{SS} 3$, and SS4 corresponding to a new, poor, very poor, and failed state of the structure.

At a first stage, this PN model does not consider the fact that the structure may be subjected to a certain functional deterioration but is still stable from a structural point of view. Consequently, in this case, maintenance can't be applied at an early time to prevent a structural failure due to functional deterioration.

In Fig. 4, P1 is the new state in which there is no scouring (FS1) and the structure is stable (SS1). Places P12 - P14 represents the functional degradation states (FS2, FS3, and FS4) and P2 $\mathrm{P} 4$ corresponds to the structural degradation states (SS2, SS3, and SS4). Transitions T12 - T14 are responsible for linking the functional degradation states and T1 - T3 link structural degradation states.

\subsection{Deterioration, inspection, and maintenance processes}

The deterioration process is a combination between functional and structural deterioration. If T12 fires before T1, the system will move to states SS2 and FS2 due to a functional failure. On the other hand, if $\mathrm{T} 1$ fires before T12, the system is subjected to a structural failure first. Upon firing of T13, scouring reaches $40 \%$ of the foundations width. This will influence the structural stability and the system will move from state SS2 to state SS3. Similarly, when T14 fires, more than half of the foundation is scoured and the system moves from a very poor state to a failed state SS4. Note that $\mathrm{T} 13$ and $\mathrm{T} 14$ are reset transition. Upon firing, they remove the tokens in $\mathrm{P} 2$ and $\mathrm{P} 3$ respectively.

Regarding inspection process, it has the same algorithm as that explained in Section 3.2. Concerning maintenance operations, minor maintenance is applied when the system is revealed to be in a poor state. In this case, reparation is done only for functional deterioration in which the scoured area is refilled by earth material. If the system is in a very poor state, major maintenance is required in which a (e.g.) riprap (rock or concrete) is added to the scoured area to repair functional degradation and the structural problem of tilting can be repaired or prevented from becoming more worse using (e.g.) riprap, anchor ties (steel beams mortared at the end), or by constructing cantilever supports made of reinforced concrete in the worst

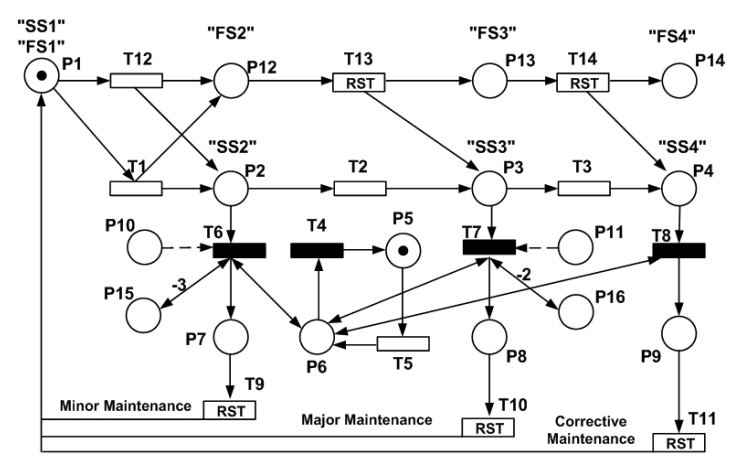

Fig. 4. PN model - Application on checkdam 
case (more expensive). When the system reaches a failed state, corrective maintenance is required in which the system should be reconstructed.

However, it is assumed that only three minor operations and two major operations can be performed before a replacement of the structure. Hence, when P15 contains three tokens, minor maintenance is inhibited until a replacement occurs. Similarly, when P16 contains two tokens, major maintenance is inhibited until corrective maintenance takes place. For this reason, when T11 fires, it resets places P15 and P16 in order to empty them from tokens. Moreover, when T9, T10, or T11 fires, a maintenance operation is carried out and resets places P12 - P14 in which the system is recovered and returns back to its new state (FS1, SS1).

The input data needed to run this model are the firing delay times associated with each transition. In this study, no historical data are available, therefor these data are assumed and are presented in Table 1.

Table 1. Transitions firing times.

\begin{tabular}{lll}
\hline Transition & $\begin{array}{l}\text { Exponential Failure } \\
\text { Rate } \lambda \\
\left(\text { year }^{-1}\right)\end{array}$ & $\begin{array}{l}\text { Constant Firing } \\
\text { Time } \\
(\text { year })\end{array}$ \\
\hline T1 & 0.2 & - \\
T2 & 0.066 & - \\
T3 & 0.2 & - \\
T12 & 0.5 & - \\
T13 & 0.1 & - \\
T14 & 0.033 & - \\
T4 T6 T7 T8 & - & 0 \\
T5 & - & 1 \\
T9 & - & 0.13 \\
T10 & - & 0.082 \\
T11 & - & 0.33 \\
\hline
\end{tabular}

With respect to the firing times of transitions linking the degraded states, it is assumed that for functional deterioration, the time taken to degrade from a state to another is increasing with the increase of deterioration. Meaning that initially, scouring will appear within a short time but its development will be slower over time. For structural deterioration, simple movement of the foundation (settlement, mild tilting) may exist at an early stage. However, a sharp tilting needs more time to occur but when it happens, failure by overturning may be triggered quickly. This explanation illustrates the variation of the firing times of T12 - T14 (functional) and T1 - T3 (structural) represented in Table 1.

The main objective of this model is to act as a decision-aiding tool. It compares different maintenance strategies to help choose the most appropriate one. This is achieved by inhibiting certain maintenance operation using places P10 and P11 in the model as explained in Section 3.4. Four different strategies are considered:

(i) Strategy 1: repair as soon as the system is not in the new state (P10 and P11 are not marked).

(ii) Strategy 2: minor maintenance is inhibited (a token is added to P10).

(iii) Strategy 3: major maintenance is inhibited (a token is added to P11).

(iv) Strategy 4: minor and major maintenance are inhibited (a token is added to P10 and P11).

\section{PN Simulation - Results \& Discussion}

The model is simulated over 100 year lifetime period of the checkdam. Since the model follows a stochastic process, many simulations are needed to obtain precise results. The number of simulations will be considered enough when convergence of results occurs. In this study, convergence is reached after 200 simulations for most of the applied strategies.

Tables 2-4 summarize the results obtained after the simulation of all maintenance strategies applied for functional and structural deterioration.

Table 2. Statistics on the average expected number of each type of maintenance operation.

\begin{tabular}{cccc}
\hline Strategy & Minor & Major & Corrective \\
\hline 1 & 11 & 6 & 3 \\
2 & 0 & 7 & 4 \\
3 & 18 & 0 & 7 \\
4 & 0 & 0 & 8 \\
\hline
\end{tabular}

Table 3. Statistics on the average sojourn time (year) in each state - Functional deterioration.

\begin{tabular}{ccccc}
\hline Strategy & FS1 & FS2 & FS3 & FS4 \\
\hline 1 & 29.63 & 58.73 & 10.05 & 0.17 \\
2 & 16.70 & 69.53 & 12.04 & 0.20 \\
3 & 33.69 & 44.13 & 20.28 & 0.40 \\
4 & 12.41 & 59.80 & 25.70 & 0.44
\end{tabular}

Table 3 and Table 4 reveal the effect of each maintenance strategy on the mean sojourn time. It is clear that when minor maintenance is prevented (strategies 2 and 4), the sojourn time of the system in the initial states SS1 and FS1 will be less than that when maintenance is applied directly when the system is no more in its new state (strategies 1 and 3). The reason behind this is that when minor 
Table 4. Statistics on the average sojourn time (year) in each state - Structural deterioration.

\begin{tabular}{cllll}
\hline Strategy & SS1 & SS2 & SS3 & SS4 \\
\hline 1 & 28.23 & 54.46 & 13.30 & 1.36 \\
2 & 15.17 & 64.80 & 15.77 & 1.70 \\
3 & 32.29 & 36.13 & 27.03 & 3.30 \\
4 & 11.02 & 50.45 & 34.00 & 4.14 \\
\hline
\end{tabular}

maintenance is disabled, the system will remain in the degraded state SS2 for a longer time. In strategy 3 , since no major maintenance is allowed, the system will reside for a long time in state SS3 $(\approx 27$ years $)$ in comparison with strategy $1(\approx 13$ year) and strategy $2(\approx 15$ years $)$. Concerning strategy 4 , only corrective maintenance is allowed, the system will reside for a long time in states SS2 and SS3 waiting for more deterioration until failure and the number of corrective maintenance will increase compared to other strategies (8 replacements).

Such results allow comparing the different maintenance strategies in terms of cost. It is assumed that the costs of minor and major operations are respectively 5\% and 50\% the cost of a replacement which is estimated to be $150,000 €$.

Having the statistics about the number of applied maintenance operations provided by the PN model, the total expected cost of each maintenance strategy is computed and given in Table 5. Fig. 5 illustrates the variation in the costs of the different maintenance operations in each strategy. It is noticed that strategy 1 is the cheapest due to the fact that the system is not allowed to deteriorate to states where expensive maintenance operations are required. It is repaired directly with minor operations (less expensive). Strategy 4 seems to be the most expensive because of the huge number of corrective maintenance in which the structure is reconstructed.

Table 5. Total maintenance strategies costs (€).

\begin{tabular}{ccccc}
\hline Strategy & Minor & Major & Corrective & Total \\
\hline 1 & 82,500 & 450,000 & 450,000 & 982,500 \\
2 & 0 & 525,000 & 600,000 & $1,125,000$ \\
3 & 135,000 & 0 & $1,050,000$ & $1,185,000$ \\
4 & 0 & 0 & $1,200,000$ & $1,200,000$ \\
\hline
\end{tabular}

\section{Conclusion}

This paper presents a methodology for modeling the behavior of checkdams when exposed to deterioration mechanisms and maintenance operations based on PNs. The PN model considers the deterioration, inspection through which the

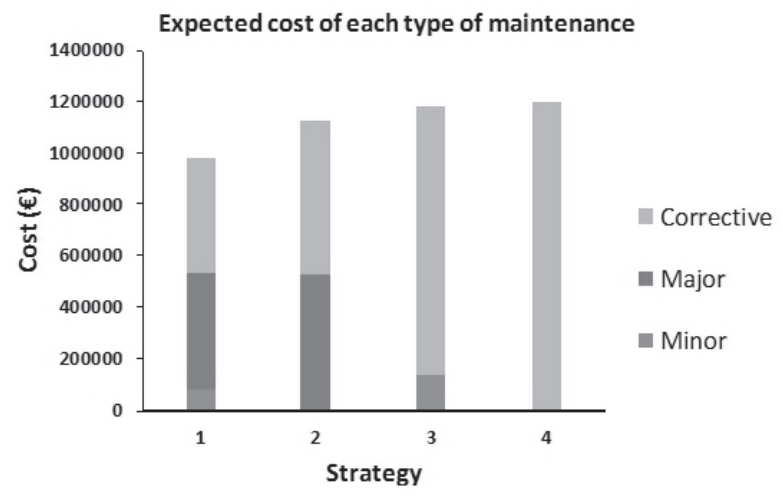

Fig. 5. Expected total cost for each maintenance operation in the four different strategies

system condition is revealed, and maintenance processes of the system. The model is simulated using Monte Carlo simulation where convergence in results is attained after 200 simulations. All processes are based on assumed input data due to the absence of real historical records. The model is used to compare different maintenance strategies in which it allows the investigation of the number of maintenance operations required, the total cost of each maintenance strategy, and the residence time of the system in each condition over its lifetime period.

PNs prove their flexibility in which they allow to simulate different maintenance strategies by simple modifications (inhibitor arcs) without changing the structure of the model. This model can support maintenance decision-making by identifying the most appropriate strategy Le and Andrews (2016). However, a comprehensive understanding of the complex deterioration process should be acquired before the calibration of the model Yianni et al. (2017).

The application done in this study is not validated due to the fact that all the input data used are assumed. The main target is to confirm the capabilities of PN model to provide helpful results supporting decision-making based on raw data and expert knowledge.

Such work can be developed further to be validated and closer to reality. Proposals involve:

- Using real historical data concerning deterioration and maintenance of torrential protection works or assessing the assumed data by experts.

- Taking into consideration maintenance operations that are not always perfect to restore the system to its initial new state.

- Studying the dependability between several chackdams constructed in series (cascade failure). 


\section{Acknowledgement}

This work is supported by the French National Research Agency in the framework of the Investissements d'Avenir program (ANR-15-IDEX02).

\section{References}

Adam, C. (1962). Kommunikation mit Automaten. Bonn: Mathematisches Institut der Universitat Bonn.

Andrews, J. (2013). A modelling approach to railway track asset management. Proceedings of the Institution of Mechanical Engineers, Part F: Journal of Rail and Rapid Transit 227(1), 56-73. cited By 41.

Arab Maki, A. and N. Shariat Zadeh (2010). Design and development of a maintenance knowledge-base system based on common kads methodology. Master's thesis, KTH, Production Engineering.

Aubry, J.-F., N. Brinzei, and M. H. Mazouni (2016, February). Systems Dependability Assessment: Benefits of Petri Net Models, Volume 1 of Systems and Industrial Engineering Series. Systems Dependability Assessment Set. ISTE Ltd and John Wiley \& Sons Inc.

Carladous, S. (2017, April). Approche intégrée d'aide à la décision basée sur la propagation de l'imperfection de l'information-application à l'efficacité des mesures de protection torrentielle. Theses, l'Ecole des Mines de SaitEtienne.

Kotzanikolaou, P., M. Theoharidou, and D. Gritzalis (2013). Cascading effects of common-cause failures in critical infrastructures. In J. Butts and S. Shenoi (Eds.), Critical Infrastructure Protection VII, Berlin, Heidelberg, pp. 171-182. Springer Berlin Heidelberg.

Le, B. and J. Andrews (2016). Petri net modelling of bridge asset management using maintenance-related state conditions. Structure and Infrastructure Engineering 12(6), 730751. cited By 4.

Le, B., J. Andrews, and C. Fecarotti (2017). A petri net model for railway bridge maintenance. Proceedings of the Institution of Mechanical Engineers, Part O: Journal of Risk and Reliability 231(3), 306-323. cited By 0.

Modarres, M. (1992). What Every Engineer Should Know about Reliability and Risk Analysis. CRC Press.

Sabaei, D., J. Erkoyuncu, and R. Roy (2015). A review of multi-criteria decision making methods for enhanced maintenance delivery. Procedia CIRP 37, 30 - 35. CIRPe 2015 - Understanding the life cycle implications of manufacturing.

Sava, A., K. Adjallah, and Z. Wang (2015). Monte carlo based petri net simulation for maintenance strategies assessment in series-parallel- series multi-physic systems. Volume 2, pp. 576-581. cited By 0.

Tacnet, J.-M., S. Carladous, C. Curt, Y. Legat, B. Rulleau, and C. Werey (2016). Combiner les modèles d'aide à la décision pour la gestion patrimoniale des infrastructures. Sciences Eaux and Territoires : la Revue du IRSTEA (20), 9097.

Yianni, P., D. Rama, L. Neves, J. Andrews, and D. Castlo (2017). A petri-net-based modelling approach to railway bridge asset management. Structure and Infrastructure Engineering 13(2), 287-297. cited By 6. 